\title{
Tracing Remnants of the Baryon Vector Current Anomaly in Neutron Radiative $\beta$-Decay
}

Susan Gardner and Daheng He

Department of Physics and Astronomy, University of Kentucky, Lexington, KY 40506-0055 USA

\begin{abstract}
We show that a triple-product correlation in the neutron radiative $\beta$-decay rate, characterized by the kinematical variable $\eta \equiv\left(\mathbf{l}_{e} \times \mathbf{l}_{v}\right) \cdot \mathbf{k}$, where $n(p) \rightarrow p\left(p^{\prime}\right)+e^{-}\left(l_{e}\right)+\bar{v}_{e}\left(l_{v}\right)+\gamma(k)$, isolates the pseudo-Chern-Simons term found by Harvey, Hill, and Hill as a consequence of the baryon vector current anomaly and $\mathrm{SU}(2)_{L} \times \mathrm{U}(1)_{Y}$ gauge invariance at low energies. The correlation appears if the imaginary part of the coupling constant is nonzero, so that its observation at anticipated levels of sensitivity would reflect the presence of sources of CP violation beyond the Standard Model. We compute the size of the asymmetry in $n \rightarrow p e^{-} \bar{v}_{e} \gamma$ decay as a function of the coupling, estimate the effect of Standard-Model final-state interactions, and discuss the role nuclear processes can play in discovering the effect.
\end{abstract}

Keywords: $\beta$-decay, decay correlations, CP violation

PACS: 23.40.-s, 11.30.Er, 12.39.Fe, 12.15.-y

\section{Introduction}

Harvey, Hill, and Hill have found that unexpected interactions involving the nucleon $N$, photon $\gamma$, and weak gauge bosons at low energies emerge from gauging the axial anomaly of QCD under the full electroweak symmetry of the Standard Model (SM) [1, 2]. In this contribution we consider how such interactions can be isolated through a triple-product momentum correlation in neutron radiative $\beta$-decay. The correlation is both parity- and naively timereversal-odd, so that it vanishes in the Standard Model save for effects induced by final-state interactions (FSI). Nevertheless, the correlation can be generated by sources of CP violation beyond the Standard Model, and such couplings, being spin-independent, are not constrained by the nonobservation of the neutron electric dipole moment (EDM). We consider the sorts of limits on its strength which can be determined at existing and anticipated facilities, as well as the size of induced correlations from known FSI. We also briefly consider the possibility of nuclear $\beta$-decay studies as well as the prospects for muon-induced reaction studies.

\section{Anomalous interactions at low energies}

The study of the low-energy spectrum of quantum chromodynamics (QCD) reveals light, pseudoscalar mesons which we interpret as the Nambu-Goldstone bosons of a spontaneously broken chiral symmetry, so that in two-flavor QCD, e.g., $\mathrm{SU}(2)_{L} \times \mathrm{SU}(2)_{R} \rightarrow \mathrm{SU}(2)_{V}$ [3, 4]. A chiral theory of mesons built on this symmetry is incomplete; the explicit inclusion of the axial anomaly, vis-a-vis the Wess-Zumino-Witten (WZW) term [5, 6], is required in order to describe observed processes such as $K \bar{K} \rightarrow 3 \pi$ and $\pi^{0} \rightarrow \gamma \gamma$ [7]. The latter reflects the nonconservation of the axial current and hence the presence of the axial anomaly [8, 9]. If we study the gauge invariance of the WZW term in 


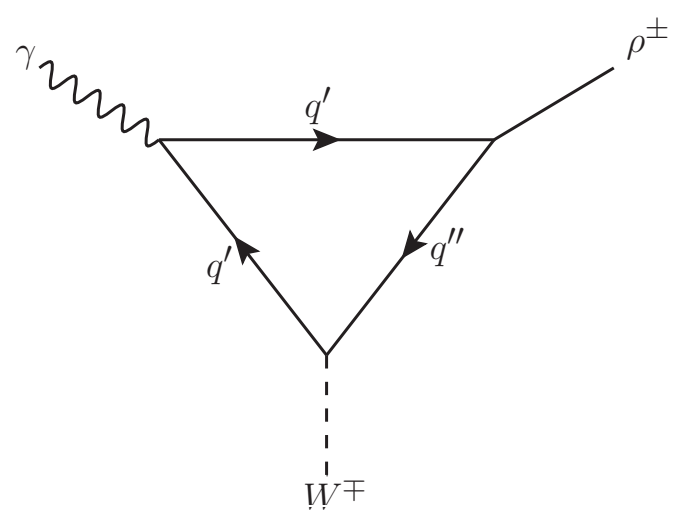

Figure 1: A possible contribution to a pCS interaction at low energy.

quantum electrodynamics, then the vector current is conserved [9, 10]. This changes, however, once we consider the gauge invariance of the WZW action under the full electroweak gauge group of the SM; the vector current is no longer conserved. Namely, in the presence of spin-one fields which couple to baryon number, the baryon vector current is anomalous and new contact interactions of pseudo-Chern-Simons form result [1, 2]. Promoting these spin-one fields to a description of the light vector mesons of QCD, $\omega$ and $\rho^{ \pm, 0}$, Harvey, Hill, and Hill recover interactions of the form $\varepsilon^{\mu \nu \rho \sigma} \omega_{\mu} Z_{v} F_{\rho \sigma}$ and $\varepsilon^{\mu \nu \rho \sigma} \rho_{\mu}^{ \pm} W_{v}^{\mp} F_{\rho \sigma}$, e.g., where $F_{\rho \sigma}$ is the electromagnetic tensor, and term them pseudo-Chern-Simons (pCS) interactions [1, 2].

Such pCS interactions can also be recovered in a chiral Lagrangian framework without explicit reference to vector mesons. In a theory of nucleons and pions with a complete set of electroweak gauge fields, treated as external sources, they appear at next-to-next-to-leading order $\left(\mathrm{N}^{2} \mathrm{LO}\right)$ in the chiral expansion [11]. To connect to the earlier analysis, the terms expressed in $\omega$ and $\rho$ degrees of freedom contribute to certain low-energy constants of the chiral Lagrangian and could well "saturate" the values of these coefficients [12]. However, dynamics involving the $\Delta$ resonance also contribute and are numerically important in the neutral-current sector [11]. At the lowest energies the effective action takes the form [1]

$$
S_{\mathrm{HHH}}=\sum_{f} \xi \int d^{4} x \epsilon^{\mu \kappa \rho \sigma} \bar{N} \gamma_{\mu} N \bar{v}_{L}^{f} \gamma_{\kappa} \nu_{L}^{f} F_{\rho \sigma} ;
$$

we adopt its charged-current analogue for our studies in $\beta$-decay. Working in the $\mathrm{SM}$ and asserting vector-meson dominance one expects $\xi \equiv \xi_{\omega}=\left(N_{c} / 12 \pi^{2}\right)\left(g_{\omega}^{2} / m_{\omega}^{2}\right)\left(e G_{F} / \sqrt{2}\right)$ [1]. These pCS interactions could play a role in a swath of processes. Possibilities include the interpretation of the low-energy event excess measured by the MiniBooNE collaboration [1, 13], as well as that of parity-violating observables in reactions such as $n p \rightarrow d \gamma$ [1, 14]. We consider the simplest possible system which can reveal the effect: neutron radiative $\beta$-decay [15].

Generally the baryon vector current is anomalous in theories in which the gauge fields couple differently to $L$ and $R$-handed quarks, so that we suppose the contact interactions discussed by Harvey, Hill, and Hill possess broader support. For example, they ought exist in theories beyond the $\mathrm{SM}$ which possess a $\mathrm{SU}(2)_{L} \times \mathrm{U}(1)_{Y}$ gauge symmetry at low energy. This notion is not very restrictive given current empirical constraints on extended gauge sectors from precision electroweak measurements and from flavor physics, note Ref. [16] for a review of these in the context of technicolor models.

The study of radiative $\beta$-decay can yield direct constraints on $\operatorname{Im} \xi$; thus we are searching for evidence of new sources of CP violation in remnants of the baryon vector current anomaly at low energy. The coupling between new matter and the gauge particles can be complex; we can then obtain a nontrivial value of $\operatorname{Im} \xi$ through the interference of the SM contribution with processes such as that illustrated in Fig. 1, where the new particles $q^{\prime}$ and $q^{\prime \prime}$ are connected by isospin raising or lowering operators. The new-matter candidates include, e.g., "quirks" [17, 18, 19, 20, 21] or “dark quarks" [22]. Interestingly "quirk" models can have novel collider signatures as well [21, 23]. 


\section{Isolating a pCS interaction in neutron radiative $\beta$-decay}

The $\varepsilon^{\mu \nu \rho \sigma}$ structure of the pCS interaction suggests its symmetry properties can be used to isolate it. In $n(p) \rightarrow$ $p\left(p^{\prime}\right)+e^{-}\left(l_{e}\right)+\bar{v}_{e}\left(l_{v}\right)+\gamma(k)$ decay the interference of the pCS term with the usual $V-A$ terms in the absolute square of the transition matrix element yields, to leading order in $\xi$,

$$
|\mathcal{M}|_{\mathrm{pCS}}^{2}=-64 M M^{\prime} \frac{e^{2} G_{F}^{2}}{2} \operatorname{Im} \bar{\xi} \frac{E_{e}}{l_{e} \cdot k}\left(\mathbf{l}_{e} \times \mathbf{l}_{v}\right) \cdot \mathbf{k}+\ldots,
$$

where $\bar{\xi} \equiv \xi /\left(e G_{F} / \sqrt{2}\right)$. The pseudo-T-odd interference term is finite as the photon energy $\omega \equiv|\mathrm{k}| \rightarrow 0$, so that its appearance is compatible with Low's theorem [24] as expected. Defining $\eta \equiv\left(\mathbf{l}_{e} \times \mathbf{I}_{v}\right) \cdot \mathbf{k}$ we partition the $\beta$-decay phase space into regions of definite sign to form an asymmetry:

$$
\mathcal{A}\left(\omega^{\min }\right) \equiv \frac{\Gamma_{\eta>0}-\Gamma_{\eta<0}}{\Gamma_{\eta>0}+\Gamma_{\eta<0}}
$$

where $\omega^{\min }$ is the lowest detectable photon energy. We recall that the usual bremstrahlung contribution is infrared sensitive [25, 26]. Working to leading recoil order and to linear order in $\bar{\xi}$, employing the parameters and formulae of Ref. [26], we find

$$
\mathcal{A}\left(\omega^{\min }=0.035 \mathrm{MeV}\right)=-2.60 \cdot 10^{-3} \operatorname{Im} \bar{\xi}\left(\mathrm{MeV}^{-2}\right), \operatorname{Br}\left(\omega^{\min }=0.035 \mathrm{MeV}\right)=1.83 \cdot 10^{-3}
$$

and

$$
\mathcal{A}\left(\omega^{\min }=0.3 \mathrm{MeV}\right)=-1.34 \cdot 10^{-2} \operatorname{Im} \bar{\xi}\left(\mathrm{MeV}^{-2}\right), \operatorname{Br}\left(\omega^{\min }=0.3 \mathrm{MeV}\right)=8.62 \cdot 10^{-5},
$$

where $\omega^{\max }=0.782 \mathrm{MeV}$. In neutron radiative $\beta$-decay the branching ratio with increasing photon energy quickly becomes phase-space limited. Nevertheless, since the number of neutrons needed to measure a significant effect is controlled by $\mathcal{A}^{2} \mathrm{Br}$, the use of photons with energies in excess of $0.3 \mathrm{MeV}$ would be more efficacious. Of course $\operatorname{Im} \bar{\xi}$ in the SM is zero, but if $\operatorname{Im} \bar{\xi} \approx \bar{\xi}_{\rho}$ the asymmetry would nevertheless be very small indeed. Employing vector-meson dominance and adopting $g_{\omega}$ and $g_{\rho}$ from a phenomenological analysis of nucleon-nucleon $(N N)$ phase shifts [27] yields $\bar{\xi}_{\rho} \approx 4.6 \times 10^{-7} \mathrm{MeV}^{-2}$ for the charged-current process, to be compared with $\bar{\xi}_{\omega} \approx 4.1 \times 10^{-6} \mathrm{MeV}^{-2}$ for the neutral-current process. To estimate the ability to detect an asymmetry we consider the counting rates from the neutron radiative $\beta$-decay experiment at NIST [28, 29]. The $e p$ double coincidence counting rate in that experiment was $20 \mathrm{~s}^{-1}$ for a quoted neutron flux density of $1.1 \times 10^{8} \mathrm{~cm}^{-2} \mathrm{~s}^{-1}$ [30]. Noting that the total available neutron rate at the NG-6 end station at NIST is actually some 20 times larger [30], then in one week of running time with $\omega^{\min }=0.3 \mathrm{MeV}$ one could have a statistical error on the asymmetry of $\sim 7 \times 10^{-3}$. Both the incident flux and the usable beam size are projected to be significantly larger in the planned New Guide Hall [30]; the estimated statistical reach on the asymmetry could then approach $\sim 1 \times 10^{-3}$. Although the neutron measurements would probe regions of model parameter space which are unconstrained by other data, it is prudent to consider the triple-product momentum correlation in nuclear radiative $\beta$ decays as well. We note in contradistinction that EDM limits impose constraints on chirality-changing operators [31]. The asymmetries will generally be larger in nuclear decays because the asymmetry grows with the energy released in the decay. In addition, since the pCS interaction engenders parity violation but does not involve the nucleon spin, it is coherent at the amplitude level [11]. There are different possibilities for its study in nuclei. As an example, in ${ }^{19} \mathrm{Ne}$ decay the energy release is some $\sim 2.7 \mathrm{MeV}$, and the ${ }^{19} \mathrm{Ne}$ lifetime is $17.2 \mathrm{~s}$. Perhaps one could study ${ }^{19} \mathrm{Ne}$ $\rightarrow{ }^{19} \mathrm{~F}$ radiative $\beta$-decay in an atom trap experiment, note Ref. [32] for a discussion of related work. An accelerator experiment might also allow access to the triple product momentum correlation; the beam momentum could define one of the needed momenta, so that one could access the correlation via a forward-backward asymmetry. The nuclear studies could possibly be realized at a rare isotope accelerator such as the Facility for Rare Isotope Beams (FRIB) at Michigan State University. Irrespective of these experimental choices, the best candidate nuclear radiative $\beta$-decay would have a large energy release and be mediated by a pure Fermi transition.

\section{Induced Correlations from Known FSI}

As long known, $O(\alpha)$ radiative corrections can mimic pseudo-T-odd correlations [33, 34] in particle decay and production processes. In neutron radiative $\beta$-decay such effects are operative as well. A similar calculation has been 


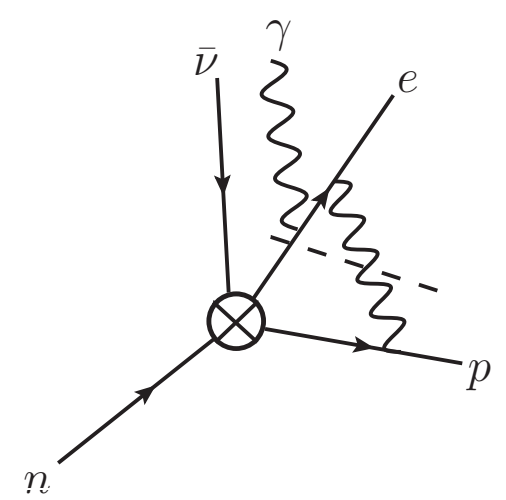

Figure 2: One contribution to the discontinuity across the $\gamma e$ cut.

performed in kaon radiative $\beta$-decay [35, 36, 37]. In neutron radiative $\beta$-decay there are three basic types of physical cuts, and the total number of cut diagrams is 14. One particular diagram associated with the $\gamma e$ cut is shown in Fig. 2 . Using the Cutkosky rules [38], we can compute the discontinuity across each physical cut. Namely, for the $\gamma e$ cut, we have

$$
-i \operatorname{Disc} \mathcal{M}^{\gamma e}(n \rightarrow p e \bar{v})=\sum_{f} \int \frac{d^{4} l_{1} d^{4} l_{2}}{(2 \pi)^{2}} \delta^{(4)}\left(l_{1}+l_{2}-l_{e}-k\right) \delta^{(+)}\left(l_{1}^{2}-m^{2}\right) \delta^{(+)}\left(l_{2}^{2}\right) \mathcal{M}^{*}(\gamma e \rightarrow \gamma e) \mathcal{M}(n \rightarrow p \bar{v} e \gamma)
$$

where $\delta^{(+)}\left(l^{2}-m^{2}\right)$ means that a particle of momentum $l$ and mass $m$ has been put on its positive energy shell. We have determined that the first nontrivial contribution to the triple momentum correlation is in radiative recoil order, which is encouraging. A complete calculation of the effect induced by the $O(\alpha)$ electromagnetic correction is in progress [15].

\section{Other Processes: Radiative Muon Capture}

Both radiative (RMC) and ordinary muon capture (OMC) on the proton can be used to study the pseudoscalar coupling $g_{p}$ of the nucleon. The value of $g_{p}$ is also predicted by the partially conserved axial current hypothesis (PCAC), which fits under the aegis of modern (heavy-baryon) chiral perturbation theory (HBChPT) [39, 40]. After much controversy, there has been significant experimental progress: the value of $g_{p}$ recently determined by the MuCap collaboration agrees with the HBChPT prediction [41, 42]. Nevertheless, disagreements of the old OMC and RMC results with theory and with each other persist [42]. We note that the pCS interaction proposed by Harvey, Hill, and Hill in the SM would contribute exclusively to RMC, so that if is of the proper numerical size and if the ortho-para transition rate in muonic molecular hydrogen is described well by theory, making the old OMC result consistent with MuCap, then all the residual discrepancies could be reasonably resolved. The numerical size of the pCS term in this context is under evaluation [43]. A plurality of effects could influence the value of $g_{P}$ extracted from RMC; notably contributions from $\Delta$ resonance degrees of freedom have been evaluated and are too small to explain the discrepancy [44].

\section{Summary}

Harvey, Hill, and Hill suggest that remnants of the baryon vector current anomaly in the Standard Model exist in low-energy interactions [1, 2]. We have argued that the new contact interactions they discuss can also appear in theories beyond the $\mathrm{SM}$ with $\mathrm{SU}(2)_{L} \times \mathrm{U}(1)_{Y}$ electroweak symmetry at low energies. We have studied how new sources of $\mathrm{CP}$ violation connected to such interactions can be probed through a triple-product momentum correlation in neutron radiative $\beta$-decay. The pCS interaction does not involve the nucleon spin; the constraints offered through the study of the pseudo-T-odd, P-odd asymmetry in neutron (or nuclear) radiative $\beta$-decay are complementary but distinct from those from EDMs.

[1] J. A. Harvey, C. T. Hill, R. J. Hill, Anomaly Mediated Neutrino-Photon Interactions at Finite Baryon Density, Phys. Rev. Lett. 99 (2007) 261601. 
[2] J. A. Harvey, C. T. Hill, R. J. Hill, Standard model gauging of the Wess-Zumino-Witten term: Anomalies, global currents, and pseudo-ChernSimons interactions, Phys. Rev. D 77 (2008) 085017.

[3] Y. Nambu, Quasi-Particles and Gauge Invariance in the Theory of Superconductivity, Phys. Rev. 117 (1960) 648.

[4] J. Goldstone, Field Theories with Superconductor Solutions, Nuovo Cimento 19 (1961) 154.

[5] J. Wess, B. Zumino, Consequences of anomalous Ward identities, Phys. Lett. B 37 (1971) 95.

[6] E. Witten, Current algebra, baryons, and quark confinement, Nucl. Phys. B223 (1983) 433.

[7] E. Witten, Global aspects of current algebra, Nucl. Phys. B223 (1983) 422.

[8] J. S. Bell, R. Jackiw, A PCAC puzzle: $\pi^{0} \rightarrow \gamma \gamma$ in the $\sigma$-model, Nuovo Cimento A 60 (1969) 47.

[9] S. L. Adler, Axial-Vector Vertex in Spinor Electrodynamics, Phys. Rev. 177 (1969) 2426.

[10] W. A. Bardeen, Anomalous Ward Identities in Spinor Field Theories, Phys. Rev. 184 (1969) 1848.

[11] R. J. Hill, Low energy analysis of $v N \rightarrow v N \gamma$ in the standard model, Phys. Rev. D 81 (2010) 013008.

[12] G. Ecker, J. Gasser, A. Pich, E. de Rafael, The Role oF Resonances in Chiral Perturbation Theory, Nucl. Phys. B321 (1989) 31.

[13] R. J. Hill, On the single photon background to $v_{e}$ appearance at MiniBooNE, arXiv:1002.4215 1.

[14] R. J. Hill, Some new implications of the anomalous baryon current in the standard model, arXiv:0806.3673 1.

[15] S. Gardner, D. He, in preparation.

[16] C. T. Hill and E. H. Simmons, Strong dynamics and electroweak symmetry breaking, Phys. Rep. 381 (2003) 235 [(Erratum-ibid. 390 (2004) 553].

[17] L. B. Okun, Thetons, JETP Lett. 31 (1980) 144

[18] L. B. Okun, Theta Particles, Nuclear Physics B 173 (1980) 1.

[19] J. D. Bjorken, Elements of Quantum Chromodynamics, SLAC Report No. SLAC-PUB-2372 (1979).

[20] S. Gupta, H. R. Quinn, Heavy quarks and perturbative quantum-chromodynamic calculations, Phys. Rev. D 25 (1982) 838.

[21] J. Kang, M. A. Luty, Macroscopic strings and "quirks" at colliders, J. High Energy Phys. 11 (2009) 065.

[22] M. Blennow, B. Dasgupta, E. F. Martinez, N. Rius, Aidnogenesis via Leptogenesis and Dark Sphalerons, arXiv:1009.3159 1.

[23] R. Harnik and T. Wizansky, Signals of New Physics in the Underlying Event, Phys. Rev. D 80 (2009) 075015.

[24] F. E. Low, Bremsstrahlung of Very Low-Energy Quanta in Elementary Particle Collisions, Phys. Rev. 110 (1958) 974.

[25] Y. V. Gaponov, R. U. Khafizov, Study of radiative neutron $\beta$ decay, Phys. Atom. Nucl. 59 (1996) 1213.

[26] V. Bernard, S. Gardner, U.-G. Meißner, C. Zhang, Radiative neutron $\beta$-decay in effective field theory, Phys. Lett. B 593 (2004) 105 [(Erratumibid. B 599 (2004) 348].

[27] R. Machleidt, K. Holinde, and Ch. Elster, The Bonn Meson-Exchange Model for the Nucleon-Nucleon Interaction, Phys. Rept. 149 (1987) 1.

[28] J. S. Nico et al., Observation of the radiative decay mode of the free neutron, Nature 444 (2006) 1059

[29] R. L. Cooper et al., Radiative $\beta$ decay of the free neutron, Phys. Rev. C 81 (2010) 035503.

[30] J. S. Nico, private communication.

[31] M. Pospelov and A. Ritz, Electric dipole moments as probes of new physics, Annals Phys. 318 (2005) 119

[32] F. Shimizu, K. Shimizu, H. Takuma, Laser cooling and trapping of Ne metastable atoms, Phys. Rev. A 39 (1989) 2758.

[33] C. G. Callan, Jr., S. B. Treiman, Electromagnetic Simulation of $T$ Violation in Beta Decay, Phys. Rev. 162 (1967) 1494.

[34] L. B. Okun and I. B. Khriplovich, $C$ odd correlation in the $K_{\mu 3}^{0}$ decay and the pion electromagnetic form factor, Sov. J. Nucl. Phys. 6 (1968) 598.

[35] V. V. Braguta, A. A. Likhoded, A. E. Chalov, T-odd correlation in the $K_{l 3 \gamma}$ decay, Phys. Rev. D 65 (2002) 054038

[36] E. H. Müller, B. Kubis, U.-G. Meißner, $T$-odd correlations in radiative $K_{l 3}^{+}$decays and chiral perturbation theory, Eur. Phys. J. C 48 (2006) 427.

[37] I. B. Khriplovich and A. S. Rudenko, $K_{l 3 \gamma}^{+}$decays revisited: branching ratios and $T$-odd momenta correlations, arXiv:1012.0147 2.

[38] R. E. Cutkosky, Singularities and discontinuities of Feynman amplitudes, J. Math. Phys. 1 (1960) 429.

[39] V. Bernard, N. Kaiser, U.-G. Meißner, QCD accurately predicts the induced pseudoscalar coupling constant, Phys. Rev. D 50 (1994) 6899.

[40] N. Kaiser, Induced pseudoscalar form factor of the nucleon at two-loop order in chiral perturbation theory, Phys. Rev. C 67 (2003) 027002.

[41] V. Andreev et al., Measurement of the rate of muon capture in hydrogen gas and the determination of the proton's pseudoscalar coupling $g_{p}$, Phys. Rev. Lett. 99 (2007) 032002.

[42] P. Winter, Muon Capture at PSI, published in these proceedings, arXiv:1012.3859v1, note Fig. 1.

[43] S. Gardner, D. He, in preparation.

[44] V. Bernard, T. R. Hemmert, U.-G. Meißner, Ordinary and radiative muon capture on the proton and the pseudoscalar form-factor of the nucleon, Nucl. Phys. A686 (2001) 290. 